\title{
Synthesis of imidazolium salt-terminated poly(amidoamine)-typed POSS-core dendrimers and their solution and bulk properties
}

\author{
Kensuke Naka ${ }^{1}$, Ryusuke Shinke ${ }^{1}$, Maki Yamada ${ }^{1}$, Fadila Djouadi Belkada ${ }^{1}$, Yoko Aijo ${ }^{1}$, Yasuyuki Irie ${ }^{1}$, \\ Sonu Ram Shankar ${ }^{2}$, Kumar Sai Smaran ${ }^{2}$, Noriyoshi Matsumi ${ }^{2}$, Shogo Tomita ${ }^{3}$ and Shinichi Sakurai ${ }^{3}$ \\ Imidazolium bromide-terminated first and second generation poly(amidoamine) (PAMAM)-typed polyhedral oligomeric \\ silsesquioxane (POSS)-core dendrimers, denoted as POSS-PIm16Br and POSS-PIm32Br, respectively, were prepared by the \\ reaction of the corresponding imidazole-terminated PAMAM-typed POSS-core dendrimers with 1-bromopropane. The structures \\ of POSS-PIm16Br and POSS-PIm32Br were identified using multinuclear nuclear magnetic resonance data, MALDI-TOF-MS \\ spectra, and elemental analysis. Wide-angle X-ray scattering (WAXS) data for cast films of the imidazolium hexafluorophosphate \\ $\left(\mathrm{PF}_{6}\right)$-terminated first and second generation POSS-core dendrimers, denoted as POSS-PIm16 $\mathrm{PF}_{6}$ and POSS-PIm32PF 6 , \\ respectively, which were prepared by anion exchange reactions of POSS-PIm16Br and POSS-PIm32Br, were characterized \\ by the occurrence of two amorphous halos, with a strong peak at $q=15 \mathrm{~nm}^{-1}$ and another weak peak around $q=10 \mathrm{~nm}^{-1}$. \\ Our present X-ray diffraction data for the cast films of POSS-PIm16PF 6 and POSS-PIm32PF 6 reflect the same features \\ that were described for 1-butyl-3-methylimidazolium hexafluorophosphate. Ionic conductivities for the imidazolium \\ bis(trifluoromethanesulfonyl)imide (TFSI)-terminated POSS-core dendrimers, which were prepared by anion exchange reactions \\ of POSS-PIm16Br and POSS-PIm32Br, were estimated by the ac impedance method after the addition of LiTFSI. Maximum \\ ionic conductivity of $4.03 \times 10^{-5} \mathrm{~S} \mathrm{~cm}^{-1}$ at $51{ }^{\circ} \mathrm{C}$ was observed for the POSS-core dendrimer of lower generation and \\ less lithium salt addition.
}

Polymer Journal (2014) 46, 42-51; doi:10.1038/pj.2013.60; published online 3 July 2013

Keywords: coordination; dendrimer; imidazolium; POSS; silsesquioxane; WAXS

Ionic liquids are organic salts with low melting temperatures that have attracted significant research interest because of their characteristic properties including high ionic conductivity, high polarity, high density, high heat capacity, and high thermal and chemical stability. ${ }^{1-3}$ An advantage of using these ionic liquids is their ability to dissolve a wide range of organic and ionic compounds. A number of strategies have emerged for using ionic liquids in advanced materials applications, including the development of ion gels composed of a polymer network swollen with ionic liquid solvents. ${ }^{2,4-8}$ A common problem encountered with both liquid electrolytes and ion gels is the propensity for electrolyte leakage from these systems. To overcome this drawback, several groups have prepared polymerized ionic liquids and their polymers as a new class of polyelectrolyte that is valuable for a number of engineering applications. Polymerized ionic liquids are emerging as a new class of materials with potential applications not only as polymeric electrolytes ${ }^{9,10}$ but as catalytic membranes, ${ }^{11}$
$\mathrm{CO}_{2}$-absorbing materials ${ }^{12-13}$ and microwave absorbing materials. ${ }^{14}$ Most examples of poly(ionic liquid)s have been linear side-chain homopolymers or copolymers. Simple polymerization of these ionic liquid monomers, however, usually leads to very poor ionic conductivity due to a considerable increase in the glass transition temperature and a reduction in the number of mobile ions after covalent bonding of the component ions.

Recent studies of the ionic conductivity of polymerized ionic liquids have demonstrated that materials must have low glass transition temperatures to obtain high levels of ionic conductivity. However, these conductive materials with low $T_{\mathrm{g}}$ are of limited use at ambient temperature in many electrochemical devices due to a lack of mechanical strength. To overcome this limitation, phase-separated block copolymers consisting of ionic and hydrophobic blocks have attracted increased attention in the design of polymer electrolytes for electrochemical devices, in which the ionic domains form continuous

${ }^{1}$ Department of Chemistry and Materials Technology, Graduate School of Science and Technology, Kyoto Institute of Technology, Kyoto, Japan; ${ }^{2}$ School of Materials Science, Japan Advanced Institute of Science and Technology (JAIST), Ishikawa, Japan and ${ }^{3}$ Department of Biobased Materials Science, Graduate School of Science and Technology, Kyoto Institute of Technology, Kyoto, Japan

Correspondence: Professor K Naka, Department of Chemistry and Materials Technology, Graduate School of Science and Technology, Kyoto Institute of Technology, Goshokaido-cho, Matsugasaki, Sakyo-ku, Kyoto 606-8585, Japan.

E-mail: kenaka@kit.ac.jp

Received 30 April 2013; revised 14 May 2013; accepted 14 May 2013; published online 3 July 2013 
paths for ion conduction and the hydrophobic domains form a physically cross-linked nonconducting matrix phase. ${ }^{15-16}$ Although block copolymers containing polymerized ionic liquids are attractive materials, several disadvantages still remain. Ordered phaseseparation sometimes requires long annealing times and depends on temperature. To apply these materials to electronic devices, adhesive properties and interfacial resistance are important. However, surface structures, which should be important for surface conductivity on electrodes, are not significantly controlled by the selfassembly system. Owing to the limited radius of gyration for block copolymers, nano-sized assemblies less than $10 \mathrm{~nm}$ are not easily achieved. To overcome this drawback, a different concept for poly(ionic liquid)s should be developed.

The use of a polyhedral oligomeric silsesquioxane (POSS)-core for dendrimer synthesis is particularly attractive because of the minimal number of required synthetic steps and their polyhedral structures can produce spherically symmetric dendrimers, even with earlier generations, more successfully than conventional cores can. The POSScore dendrimers have relatively globular conformations and few entanglements of their branches, with a high proportion of terminal functional groups positioned on the external surfaces of the dendrimers, even in earlier generations of the dendrimers. ${ }^{17-22}$ Recently, we have successfully synthesized imidazole-terminated first and second generations of poly(amidoamine) (PAMAM)-typed POSScore dendrimers. ${ }^{22}$ The terminal function groups in the first generation POSS-core dendrimer are isolated and inhibit interaction with neighboring branches, while those in the second generation dendrimer are closely positioned on the external surfaces due to fewer entanglements of their branches. In addition, PAMAM-typed POSScore dendrimers have synthetic advantages over traditional ethylenediamine core PAMAM dendrimers; the number of synthetic steps is minimized, and reaction time is significantly reduced due to fewer terminal entanglements. These aspects indicate that POSS-core dendrimers terminated with ionic liquids are an ideal candidate for the construction of ordered nano-sized network architectures for solid ionic conductive materials. As the POSS-core dendrimers have globular conformations due to their rigid silsesquioxane core, their three-dimensional structures would be maintained in the solid state. The high proportion of terminal functional groups positioned on the external surfaces of the dendrimers caused terminal ionic liquid domains to form continuous molecular-level network paths for ion conduction. Molecular-level phase separation $\sim 2 \mathrm{~nm}$ can be achieved, because of the well-controlled design of the dendrimer structures. The most significant advantage of the present system is that the terminal ionic liquid units may be exposed to the surface of the dendrimer assemblies, meaning that continuous molecular-level network paths for ion conduction effectively form from one side of the interface to the other. Here, we have synthesized imidazolium saltterminated POSS-core PAMAM-typed dendrimers and studied their solution and bulk properties and polyelectrolyte functions. There have been a few previous pioneering reports about ionic liquid-like unit-terminated dendrimers and hyperbranched polymers. ${ }^{23-26}$ Chujo and Co-workers ${ }^{23,24}$ first reported POSS-based room temperature ionic liquids that were synthesized from POSSoctacarbonyl anions and the imidazolium cation. Dai et al. prepared Brønsted acid-base ionic liquid-terminated PAMAM dendrimers ${ }^{25}$ that possessed air stability, hydrophobicity and high proton conductivity. However, the many entanglements of their branches made control over the expected bulk phase structure difficult. The present materials are the first examples of imidazolium salt-terminated POSS-based dendrimers.

\section{EXPERIMENTAL PROCEDURE}

\section{Materials}

All solvents and chemicals were obtained as reagent-grade quality and used without further purification. The first and second generation imidazoleterminated POSS-core PAMAM-typed dendrimers (POSS-Im16 and POSSIm32) were prepared according to the literature, ${ }^{22}$ and purified by sizeexclusion chromatography (LH-20) using methanol as an eluent.

\section{Measurements}

${ }^{1} \mathrm{H}-(400 \mathrm{MHz}),{ }^{13} \mathrm{C}-(100 \mathrm{MHz})$ and ${ }^{29} \mathrm{Si}-(100 \mathrm{MHz})$ nuclear magnetic resonance (NMR) spectra were recorded on a BRUKER PDX-300 (Bruker Biospin $\mathrm{GmbH}$, Rheinstetten, Germany). A $30^{\circ}$ pulse of $15 \mathrm{~ms}$ and a delay of $1 \mathrm{~s}$ were used for all ${ }^{1} \mathrm{H}$-NMR measurements. MALDI-TOF-MS was recorded on a BRUKER Autoflex II instrument (Bruker Daltonics, Billerica, MA, USA) using a 2.5-dihydroxybenzoic acid matrix. Fourier transform infrared spectra were obtained on a JASCO FT/IR-4100 (JASCO, Tokyo, Japan) spectrometer using $\mathrm{KBr}$ pellets. UV-vis spectra were recorded on a JASCO spectrophotometer V-670 KKN (JASCO). Differential scanning calorimetry (DSC) was recorded on a TA Instruments 2920 Modulated DSC (TA Instruments, New Castle, DE, USA). Thermogravimetric analysis (TGA) was performed on a TA Instruments Hi-Res Modulated TGA 2950 thermogravimetric analyzer (TA Instruments) under $\mathrm{N}_{2}$. Ionic conductivity was obtained using stainless steel electrodes by the complex impedance method on a Solartron model 1260 impedance analyzer (Solartron Analytical, Schlumberger, Farnborough, UK) under an ac frequency range of $1 \mathrm{~Hz}-1 \mathrm{MHz}$. During measurements, the temperature was varied from $30-60{ }^{\circ} \mathrm{C}$ using SU-221 (ESPEC, Osaka, Japan).

\section{POSS-PIm16Br}

To a DMF (24 ml) solution of POSS-Im16 (1.31 g, $0.349 \mathrm{mmol}), 24$ equivalent of 1-bromopropane $(16.5 \mathrm{~g}, 0.134 \mathrm{mmol})$ was added under nitrogen. After stirring the reaction mixture at $40^{\circ} \mathrm{C}$ for $24 \mathrm{~h}$, the volatile materials were removed under reduced pressure to obtain a colorless oil of crude POSSPIm16Br. The crude product was purified by preparative size-exclusion chromatography (LH-20) using a methanol eluent to remove the unreacted products. The yield of pure POSS-PIm16Br was $1.83 \mathrm{~g}(0.319 \mathrm{mmol}, 91 \%)$.

${ }^{1} \mathrm{H}-\mathrm{NMR}\left(\mathrm{CD}_{3} \mathrm{OD}\right): \delta 9.27$ (bs, $\left.16 \mathrm{H}\right), 7.83$ (bs, $\left.16 \mathrm{H}\right), 7.74$ (bs, $16 \mathrm{H}$ ), 4.37 (bs, 32H), $4.25(\mathrm{t}, 32 \mathrm{H}), 3.24(\mathrm{~m}, 32 \mathrm{H}), 2.97$ (bs, 32H), $2.71(\mathrm{bs}, 16 \mathrm{H})$, $2.56(\mathrm{bs}, 32 \mathrm{H}), 2.15(\mathrm{~m}, 32 \mathrm{H}), 1.94(\mathrm{~m}, 32 \mathrm{H}), 1.66(\mathrm{~m}, 16 \mathrm{H}), 0.98(\mathrm{t}, 48 \mathrm{H})$, 0.74 (bs, $16 \mathrm{H}) .{ }^{13} \mathrm{C}-\mathrm{NMR}\left(\mathrm{CD}_{3} \mathrm{OD}\right): \delta 173.55,137.54,123.85,123.73,56.77$, $52.30,50.72,48.31,36.68,32.67,31.01,30.08,24.45,20.07,11.06,10.00{ }^{29}$ $\mathrm{Si}-\mathrm{NMR}\left(\mathrm{CD}_{3} \mathrm{OD}\right): \delta-66.43$.

Anal. Calcd. for $\mathrm{C}_{216} \mathrm{~N}_{56} \mathrm{O}_{28} \mathrm{H}_{384} \mathrm{Si}_{8} 50 \mathrm{H}_{2} \mathrm{O}$ : C, 39.20; H, 7.37; N, $11.86 \%$. Found: C, 39.20; H, 6.99; N, $11.55 \%$.

\section{POSS-PIm32Br}

To a DMF $(15 \mathrm{ml})$ solution of POSS-Im32 $(0.815 \mathrm{~g}, 0.0965 \mathrm{mmol}), 24$ equivalent of 1-bromopropane $(13.9 \mathrm{~g}, 0.111 \mathrm{~mol})$ was added under nitrogen. After stirring the reaction mixture at $40^{\circ} \mathrm{C}$ for $24 \mathrm{~h}$, the volatile materials were removed under reduced pressure to obtain a colorless oil of crude POSSPIm32Br. The crude product was purified by preparative size-exclusion chromatography (LH-20) using a methanol eluent to remove the unreacted products. The yield of pure POSS-PIm32Br was $1.04 \mathrm{~g}(0.0865 \mathrm{mmol}, 90 \%)$.

${ }^{1} \mathrm{H}-\mathrm{NMR}\left(\mathrm{CD}_{3} \mathrm{OD}\right): \delta 9.25(\mathrm{~s}, 32 \mathrm{H}), 7.84(\mathrm{~s}, 32 \mathrm{H}), 7.73(\mathrm{~s}, 32 \mathrm{H}), 4.38$ $(\mathrm{t}, 64 \mathrm{H}), 4.23(\mathrm{t}, 64 \mathrm{H}), 3.23(\mathrm{~m}, 96 \mathrm{H}), 2.90(\mathrm{~m}, 96 \mathrm{H}), 2.71(\mathrm{~m}, 64 \mathrm{H}), 2.48$ $(\mathrm{m}, 64 \mathrm{H}), 2.14(\mathrm{t}, 64 \mathrm{H}), 1.91(\mathrm{~m}, 64 \mathrm{H}), 1.83(\mathrm{~m}, 16 \mathrm{H}), 0.79(\mathrm{~m}, 16 \mathrm{H})$. ${ }^{13} \mathrm{C}-\mathrm{NMR}\left(\mathrm{CD}_{3} \mathrm{OD}\right): \delta 174.64,172.90,137.55,123.83,123.73,56.87,53.36$, $52.31,51.15,50.62,48.36,38.35,36.67,34.53,32.31,31.06,24.45,20.02,11.07$, 10.10. ${ }^{29} \mathrm{Si}-\mathrm{NMR}\left(\mathrm{CD}_{3} \mathrm{OD}\right): \delta-66.74$.

Anal. Calcd. for $\mathrm{C}_{456} \mathrm{~N}_{124} \mathrm{O}_{64} \mathrm{H}_{808} \mathrm{Si}_{8} 85 \mathrm{H}_{2} \mathrm{O}$ (dendrimer missing four branches): C, 41.98 ; H, 7.56; N, 13.32\%. Found: C, 41.81; H, 7.36; N, $13.20 \%$.

\section{POSS-PIm16 $\mathrm{PF}_{6}$}

POSS-PIm16Br $(0.138 \mathrm{~g}, 0.0242 \mathrm{mmol})$ was dissolved in water $(2.8 \mathrm{ml})$. An aqueous solution $(1.8 \mathrm{ml})$ of $\mathrm{KPF}_{6}(0.107 \mathrm{~g}, 0.581 \mathrm{mmol}$, corresponding to 1.5 equivalent of the imidazolium units in POSS-PIm16Br) was added to the 
dendrimer aqueous solution. White dendrimers precipitated out of solution. The resulting precipitates were washed several times with pure water, until no residual bromide anion was detected with the use of $\mathrm{AgNO}_{3}$. The yield of POSS-PIm16PF 6 was $95 \%$.

Anal. Calcd. for $\mathrm{C}_{216} \mathrm{~N}_{56} \mathrm{O}_{28} \mathrm{H}_{384} \mathrm{Si}_{8} 19.3 \mathrm{PF}_{6} 5 \mathrm{H}_{2} \mathrm{O}: \mathrm{C}, 35.41 ; \mathrm{H}, 5.42 \mathrm{~N}$, 10.71 F, 30.03\%. Found: C, 35.26; H, 5.53; N, 10.54; F, 29.94\%.

The sample for ${ }^{1} \mathrm{H}-\mathrm{NMR}$ analysis was prepared as follows to avoid an unexpected spectrum from partial protonation of the tertiary amines in POSS-PIm16PF . POSS-PIm16Br $(0.138 \mathrm{~g}, 0.0248 \mathrm{mmol})$ was dissolved in water $(2.8 \mathrm{ml})$. The solution $\mathrm{pH}$ was adjusted to 9 by $0.1 \mathrm{M} \mathrm{NaOH}$ aqueous solution. An aqueous solution $(1.8 \mathrm{ml})$ of $\mathrm{KPF}_{6}(0.207 \mathrm{~g}, 0.581 \mathrm{mmol}$, corresponding to 1.5 equivalent of the imidazolium units) was then added to the dendrimer aqueous solution.

${ }^{1} \mathrm{H}-\mathrm{NMR}$ (acetone- $\mathrm{d}_{6}$ ): $\delta 8.99$ (bs, 16H), 7.45 (bs, 32H), $4.37(\mathrm{t}, 32 \mathrm{H}), 4.28$ $(\mathrm{t}, 32 \mathrm{H}), 3.31(\mathrm{~m}, 32 \mathrm{H}), 2.85(\mathrm{~m}, 32 \mathrm{H}), 2.56$ (bs, 16H), 2.42 (bs, 32H), 2.15 $(\mathrm{t}, 32 \mathrm{H}), 1.95(\mathrm{~m}, 32 \mathrm{H}), 1.60(\mathrm{~m}, 16 \mathrm{H}), 0.95(\mathrm{t}, 48 \mathrm{H}), 0.54(\mathrm{~m}, 16 \mathrm{H})$.

\section{POSS-PIm32 $\mathrm{PF}_{6}$}

POSS-PIm32 PF $_{6}$ was prepared in a similar manner as POSS-PIm16 PF $_{6}$. POSS-PIm32Br $(0.145 \mathrm{~g}, 0.0117 \mathrm{mmol})$ was dissolved in water $(2.8 \mathrm{ml})$. An aqueous solution $(1.8 \mathrm{ml})$ of $\mathrm{KPF}_{6}(0.104 \mathrm{~g}, 0.562 \mathrm{mmol}$, corresponding to 1.5 equivalent of the imidazolium units in POSS-PIm32Br) was added to the dendrimer aqueous solution. The yield of POSS-PIm32PF 6 was $99 \%$.

Anal. Calcd. for $\mathrm{C}_{456} \mathrm{H}_{808} \mathrm{~N}_{124} \mathrm{O}_{64} \mathrm{Si}_{8} 35 \mathrm{PF}_{6} 6 \mathrm{H}_{2} \mathrm{O}$ (dendrimer missing four branches): C, 37.88; H, 5.72 N, $12.01 \mathrm{~F}, 27.59 \%$. Found: C, 37.33; H, 5.83 N, 12.08; F, 27.63\%.

The sample for ${ }^{1} \mathrm{H}-\mathrm{NMR}$ analysis was prepared under the same conditions as those for POSS-PIm16 $\mathrm{PF}_{6}$.

${ }^{1} \mathrm{H}-\mathrm{NMR}$ (acetone- $\left.\mathrm{d}_{6}\right): \delta 9.03(\mathrm{~s}, 32 \mathrm{H}), 7.75(\mathrm{~s}, 32 \mathrm{H}), 7.66(\mathrm{~s}, 32 \mathrm{H}), 4.38$ $(\mathrm{t}, 64 \mathrm{H}), 4.29(\mathrm{t}, 64 \mathrm{H}), 3.31(\mathrm{~m}, 96 \mathrm{H}), 2.84(\mathrm{~m}, 96 \mathrm{H}), 2.63(\mathrm{~m}, 64 \mathrm{H}), 2.41$ $(\mathrm{m}, 64 \mathrm{H}), 2.15(\mathrm{t}, 64 \mathrm{H}), 1.96(\mathrm{~m}, 64 \mathrm{H}), 1.61(\mathrm{~m}, 16 \mathrm{H}), 0.95(\mathrm{t}, 96 \mathrm{H}), 0.67$ $(\mathrm{m}, 16 \mathrm{H})$.

\section{POSS-PIm16TFSI}

POSS-PIm16Br $(0.198 \mathrm{~g}, 0.0346 \mathrm{mmol})$ was dissolved in water $(2.8 \mathrm{ml})$. An aqueous solution $(1.8 \mathrm{ml})$ of lithium bis(trifluoromethanesulfonyl)imide (LiTFSI) $(0.239 \mathrm{~g}, 0.832 \mathrm{mmol}, 1.5$ equivalent of the imidazolium units in POSS-PIm16Br) was added to the dendrimer aqueous solution. White dendrimers precipitated from the solution. After keeping the dispersed solution at room temperature over-night, the precipitates were isolated by decantation, washed several times with water and dried under vacuum to obtain POSS-Im16TFSI. The yield of POSS-Im16TFSI was 99\%.

Anal. Calcd. for $\mathrm{C}_{216} \mathrm{~N}_{56} \mathrm{O}_{28} \mathrm{H}_{384} \mathrm{Si}_{8} 19\left(\mathrm{C}_{2} \mathrm{~F}_{6} \mathrm{NO}_{4} \mathrm{~S}_{2}\right) 10 \mathrm{H}_{2} \mathrm{O}$ : C, 30.64; H, 4.09 N, 10.69 F, 21.75\%. Found: C, 30.24; H, 3.96; N, 10.31; F, 21.97\%.

The sample for ${ }^{1} \mathrm{H}-\mathrm{NMR}$ analysis was prepared under the same conditions as those for POSS-PIm16 PF $_{6}$, but an aqueous solution of LiTFSI was used instead of a solution containing $\mathrm{KPF}_{6}$. A peak corresponding to the hydrogen atom at $\mathrm{C} 2$ on the imidazolium ring was missing due to its acidic character.

${ }^{1} \mathrm{H}-\mathrm{NMR}\left(\mathrm{CD}_{3} \mathrm{OD}\right): \delta 7.64(\mathrm{~s}, 16 \mathrm{H}), 4.24(\mathrm{t}, 16 \mathrm{H}), 4.17(\mathrm{t}, 32 \mathrm{H}), 3.12$ $(\mathrm{t}, 32 \mathrm{H}), 2.81(\mathrm{~m}, 32 \mathrm{H}), 2.55(\mathrm{~m}, 16 \mathrm{H}), 2.40(\mathrm{~m}, 32 \mathrm{H}), 2.08(\mathrm{~m}, 32 \mathrm{H}), 1.90$ $(\mathrm{m}, 32 \mathrm{H}), 1.59(\mathrm{~m}, 16 \mathrm{H}), 0.95(\mathrm{t}, 48 \mathrm{H}), 0.63(\mathrm{~m}, 16 \mathrm{H})$.

\section{POSS-PIm32TFSI}

POSS-PIm32TFSI was prepared in a similar manner as POSS-PIm16TFSI. POSS-PIm32Br $(0.248 \mathrm{~g}, 0.020 \mathrm{mmol})$ was dissolved in water $(2.8 \mathrm{ml})$. An aqueous solution $(1.8 \mathrm{ml})$ of LiTFSI $(0.276 \mathrm{~g}, 0.960 \mathrm{mmol}$, corresponding to 1.5 equivalent of the imidazolium units in POSS-PIm32Br) was added to the dendrimer aqueous solution. White dendrimers precipitated from solution. After keeping the dispersed solution at room temperature over-night, the precipitates were isolated by decantation, washed several times with water and dried under vacuum to obtain POSS-Im32TFSI. The yield of POSS-Im32TFSI was $99 \%$.

Anal. Calcd. for $\mathrm{C}_{456} \mathrm{H}_{808} \mathrm{~N}_{124} \mathrm{O}_{64} \mathrm{Si}_{8} 35.5\left(\mathrm{C}_{2} \mathrm{~F}_{6} \mathrm{NO}_{4} \mathrm{~S}_{2}\right.$ ) $15 \mathrm{H}_{2} \mathrm{O}$ (dendrimer missing four branches): C, 32.47; H, 4.33 N, 11.46 F, 20.76\%. Found: C, 32.45; H, 4.31; N, 11.48; F, 20.73\%.
The sample for ${ }^{1} \mathrm{H}-\mathrm{NMR}$ analysis was prepared under the same conditions as those for POSS-PIm16PF 6 , but an aqueous solution of LiTFSI was used instead of a solution containing $\mathrm{KPF}_{6}$. A peak corresponding to the hydrogen atom at $\mathrm{C} 2$ on the imidazolium ring was missing due to its acidic character.

${ }^{1} \mathrm{H}-\mathrm{NMR}\left(\mathrm{CD}_{3} \mathrm{OD}\right): \delta 7.65(\mathrm{~s}, 32 \mathrm{H}), 7.63(\mathrm{~s}, 32 \mathrm{H}), 4.24(\mathrm{t}, 64 \mathrm{H}), 4.17$ $(\mathrm{t}, 64 \mathrm{H}), 3.13(\mathrm{~m}, 96 \mathrm{H}), 2.81(\mathrm{~m}, 96 \mathrm{H}), 2.60(\mathrm{~m}, 64 \mathrm{H}), 2.38(\mathrm{~m}, 64 \mathrm{H}), 2.08$ $(\mathrm{t}, 64 \mathrm{H}), 1.91(\mathrm{~m}, 64 \mathrm{H}), 1.59(\mathrm{~m}, 16 \mathrm{H}), 0.95(\mathrm{t}, 96 \mathrm{H}), 0.62(\mathrm{~m}, 16 \mathrm{H})$.

\section{Potentiometric titration}

Potentiometric titrations were performed at room temperature with a $\mathrm{pH}$ meter (Horiba D-52, Kyoto, Japan) and glass electrode. The titrations were performed with $0.1 \mathrm{M} \mathrm{NaOH}$ in $2.0 \mathrm{M} \mathrm{NaCl}$ and a constant number of imidazole units in the dendrimers. For each aqueous solution of POSS-PIm16Br $(0.054 \mathrm{mmol})$ and POSS-PIm32Br $(0.01 \mathrm{mmol})$, which correspond to 0.43 and $0.24 \mathrm{mmol}$ of the branched tertiary amine units, respectively, in $60 \mathrm{ml}$ of distilled water with $2.0 \mathrm{M} \mathrm{NaCl}$, the $\mathrm{pH}$ was adjusted to 3 with $0.5 \mathrm{M} \mathrm{HCl}$.

\section{Photometric determination}

Aqueous $\mathrm{CuSO}_{4}$ solutions were added to aqueous dendrimer solutions that ranged in concentration ratio ([dendrimer] $/\left[\mathrm{Cu}^{2+}\right]$ ) from 0.025 to 1.0 . The concentration of the $\mathrm{CuSO}_{4}$ solution was held constant at $0.1 \mathrm{~mm}$.

\section{Wide-angle $\mathrm{X}$-ray scattering}

Wide-angle X-ray scattering (WAXS) measurements using highly brilliant synchrotron X-rays with a wavelength of $0.150 \mathrm{~nm}$ were carried out at beamline BL-9C at the Photon Factory of the High Energy Accelerator Research Organization, Tsukuba, Japan. An imaging plate, BAS-IP MS 2025 (Fuji Photo Film, Tokyo, Japan; size: $200 \times 250 \mathrm{~mm}^{2}$ ), of which the actual pixel size is $100 \times 100 \mu \mathrm{m}^{2}$, was used as a two-dimensional detector. The typical exposure time was in the range 10-30 s. BAS2500 (Fuji Photo Film) was used for the development of exposed images on the imaging plate.A polyethylene crystal was used as a standard sample for calibrating the magnitude of the scattering vector, $q$, as defined by $q=(4 \pi / \lambda) \sin (\theta / 2)$, where $\lambda$ and $\theta$ are the $\mathrm{X}$-ray wavelength and the scattering angle, respectively. The two-dimensional WAXS patterns were further converted to one-dimensional profiles by conducting the circular average. In-situ WAXS measurements at sub-ambient temperatures were conducted using Linkam LK-600 M (Japan Hightech, Fukuoka, Japan) as a cooling unit. Before each measurement at a given temperature, the sample was maintained isothermally for at least $1 \mathrm{~min}$.

\section{RESULTS AND DISCUSSION}

Synthesis and characterization of imidazolium bromide-terminated poss-core dendrimers

Imidazolium cation-terminated first and second generation PAMAMtyped POSS-core dendrimers were prepared by reacting the corresponding imidazole-terminated POSS-core dendrimers with 1-bromopropane (Schemes 1 and 2). The first and second generation imidazole-terminated PAMAM-typed POSS-core dendrimers (POSS-Im16 and POSS-Im32) were prepared and characterized in our previous report. ${ }^{22}$ According to our previous report, the ${ }^{1} \mathrm{H}-\mathrm{NMR}$ spectrum and elemental analysis of the starting second generation imidazole-terminated POSS-core dendrimer (POSS-Im32) matched those of a dendrimer missing four branches. ${ }^{22}$ When DMSO was used as a solvent for the reaction of POSS-Im32 with 1-bromopropane, the reaction proceeded as a homogeneous solution. After the solvent was removed under reduced pressure, a colorless oil of crude products remained. The ${ }^{1} \mathrm{H}-\mathrm{NMR}$ spectra of the crude products showed a peak around 3.5 p.p.m., indicating quarternarization of the branched tertiary amine groups in POSS-Im32. The integrations corresponding to the $n$-propyl groups were also larger than the expected values. These results suggest that the branched tertiary amine groups and the terminal imidazole units in the dendrimer were quarternarized. We found that the quarternarization of the tertiary 


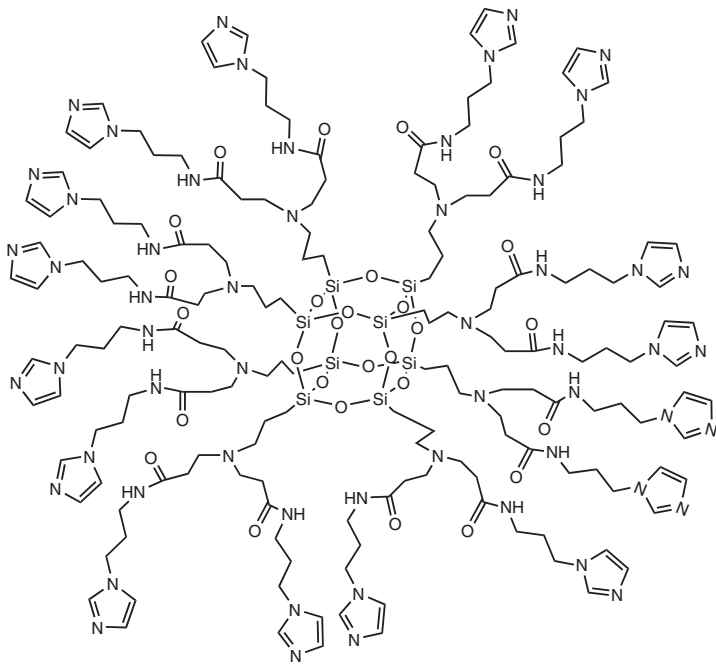

POSS-Im16

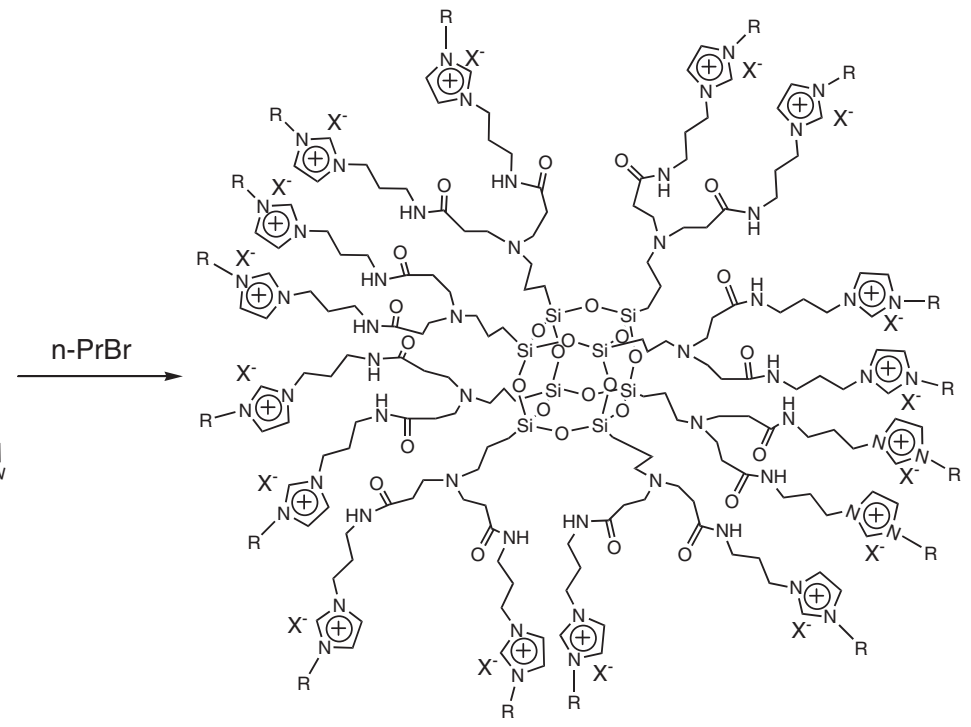

POSS-PIm16Br : $\mathrm{R}=n-\mathrm{Pr}, \mathrm{X}=\mathrm{Br}$

Scheme 1 Synthesis of POSS-PIm16Br.
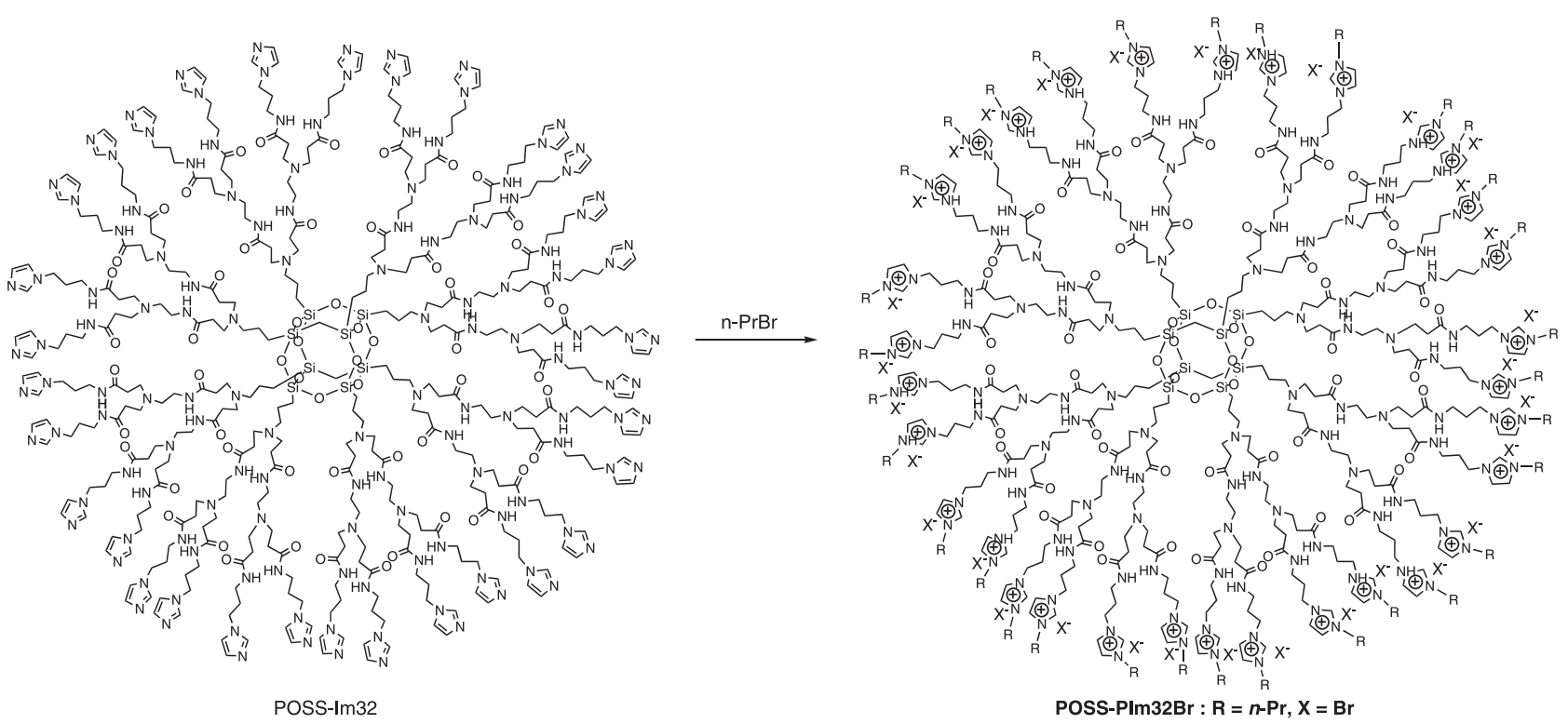

Scheme 2 Synthesis of POSS-PIm32Br.

amine groups was inhibited when the reaction proceeded in DMF. Dispersion was evident during the reaction in DMF, which may inhibit the quarternarization of the branched tertiary amine groups. After the reaction, crude oily products were separated. The crude product was purified by preparative size-exclusion chromatography (LH-20) using methanol as an eluent to remove unreacted 1-bromopropane. The obtained products were soluble in $\mathrm{H}_{2} \mathrm{O}$, methanol, ethanol, DMF and DMSO, but they were insoluble in chloroform, acetone and THF.

The structures of POSS-PIm16Br and POSS-PIm32Br were identified on the basis of multinuclear NMR and MALDI-TOF-MS spectra and elemental analysis. The degrees of functionalization of the dendrimers with the imidazolium unit were estimated using ${ }^{1} \mathrm{H}-\mathrm{NMR}$ (see Supplementary Information). Quantitative reactions of both dendrimers were supported by the integration of the ${ }^{1} \mathrm{H}-\mathrm{NMR}$ spectrum peaks corresponding to the aromatic protons on the imidazolium rings and those from the methyl protons on the $n$-propyl groups. By calculating and comparing the areas of the peaks of the aromatic protons present on the imidazole ring and the methyl protons on the $n$-propyl groups with the integrations of the methylene protons on the POSS-core as well as the remaining methoxy protons, the degrees of imidazolium terminal functionalization of POSS-PIm16Br and POSS-PIm32Br were 96 and $88 \%$, respectively. The degrees of functionalization of the starting imidazole-terminated PAMAM-typed POSS-core dendrimers (POSS-Im16 and POSS-Im32) were 98 and 90\%, respectively, providing evidence of the quantitative reactions with 1-bromopropane. The degree of functionalization of $90 \%$ for POSS-Im 32 corresponds to a dendrimer 
missing four branches. The ${ }^{13} \mathrm{C}-\mathrm{NMR}$ spectra of both dendrimers also supported their structures. In the ${ }^{29} \mathrm{Si}$ NMR spectra, peaks for the POSS units in POSS-PIm16Br and POSS-PIm32Br were observed at -66.43 and -66.74 p.p.m., respectively (see Supplementary Information). In the former spectra, minor broad peaks were detected at around -55 to -60 p.p.m., indicating possible slight decomposition of the POSS-core. In the case of POSS-PIm32Br, no other peaks were detected; thus, no decomposition of the POSS-cores. These results suggest that the increased generation numbers inhibit decomposition by basic compounds, most likely including those in the reaction solvent, by inhibiting attack to the silsesquioxane core. ${ }^{22}$ The elemental analysis for POSS-PIm16Br matched that of fully functionalized dendrimer associated with $50 \mathrm{H}_{2} \mathrm{O}$ molecules. The elemental analysis for POSS-PIm32Br matched that of a dendrimer missing four branches associated with $85 \mathrm{H}_{2} \mathrm{O}$ molecules.

MALDI-TOF-MS analyses of POSS-PIm16Br and POSS-PIm32Br showed the broad ill-defined peaks (Figure 1) that are typical for ionic high-generation dendrimers. ${ }^{27}$ A single broad peak centered around $5700 \mathrm{~m} / \mathrm{z}$ was observed for POSS-PIm16Br. This value is very close to the molecular mass $\left(5716.912 \mathrm{~g} \mathrm{~mol}^{-1}\right)$ of the fully functionalized dendrimer. These data support the quantitative quarternarization of the imidazole unit in the starting first generation imidazoleterminated POSS-core dendrimer. The mass spectrum of POSSPIm32Br showed a broad peak centered around $4700 \mathrm{~m} / \mathrm{z}$ and a shoulder at higher molecular mass in the range of $8000 \sim 12000 \mathrm{~m} / z$, which can be assigned as doubly and singly charged molecular ion peaks, respectively. The center of mass of the peak at 10000 is very close to the molecular mass $\left(11514 \mathrm{~g} \mathrm{~mol}^{-1}\right)$ of the dendrimer missing four branches. According to a previous report, MALDI-TOFMS analysis of all amine-terminated PAMAM dendrimers with an ethylenediamine core also showed peaks corresponding to both doubly and singly charged molecular ions. ${ }^{27}$

Solution behaviors of imidazolium bromide-terminated POSSCore dendrimers

The results of $\mathrm{pH}$ titrations of POSS-PIm16Br and POSS-PIm32Br in aqueous solutions are shown in Figure 2. The $\mathrm{pKa}$ values of
POSS-PIm16Br and POSS-PIm32Br are 7.8 and 6.9, respectively. These values suggest that the ammonium groups in POSS-PIm16Br were not significantly deprotonated relative to those in POSS-PIm32Br. In the case of POSS-PIm32Br, an inflection point is observed at $\mathrm{pH} 7.5$, which corresponds to a degree of deprotonation of 0.7. The total number of amine groups in POSS-PIm32Br is 24, including 8 in the first shell and 16 in the second shell. The titration result suggests that the degree of deprotonation of 0.7 matched the deprotonation of the second shell in POSS-PIm32Br. These results indicate that the deprotonation of the 16 amine groups in the second shell is easier to achieve than that of the 8 amine groups in the first shell.

The complexing behaviors of POSS-PIm16Br and POSS-PIm32Br with $\mathrm{Cu}^{2+}$ were studied. The coordination geometry of the $\mathrm{Cu}^{2+}$ complexation system is expected at the maximum absorption of the $\mathrm{d}-\mathrm{d}$ transition. Upon increasing the concentration of POSS-PIm16Br, only 1 weak and broad absorption band was observed at $\sim 800 \mathrm{~nm}$ (Figure 3a), which represents the $\mathrm{Cu}^{2+} \mathrm{d}-\mathrm{d}$ transition for $\left[\mathrm{Cu}\left(\mathrm{H}_{2} \mathrm{O}\right)_{6}\right]^{2+}$ in a tetragonally distorted octahedral ligand field. ${ }^{28-33}$ This observation suggests that no coordination with $\mathrm{Cu}^{2+}$ occurred in POSS-PIm16Br. In the case of the carboxylic acid-terminated first generation PAMAM-typed POSS-core

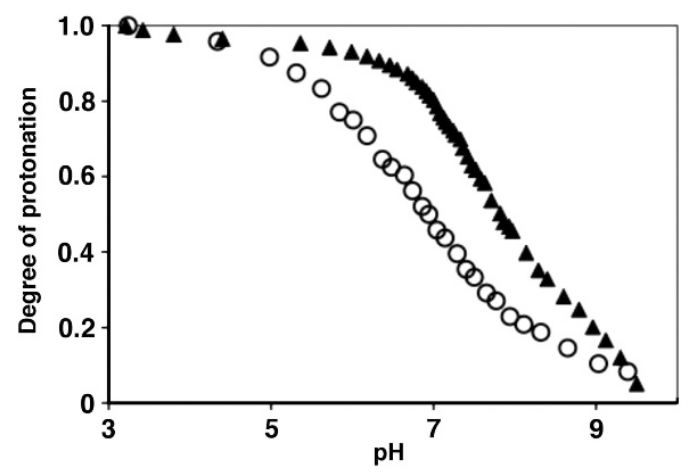

Figure 2 Potentiometric titrations of POSS-PIm16Br ( $\mathbf{\Delta})$ and POSS$\mathrm{PIm} 32 \mathrm{Br}(\mathrm{O})$ in $2.0 \mathrm{M} \mathrm{NaCl}$ solution with $0.1 \mathrm{~m} \mathrm{NaOH}$ solution.
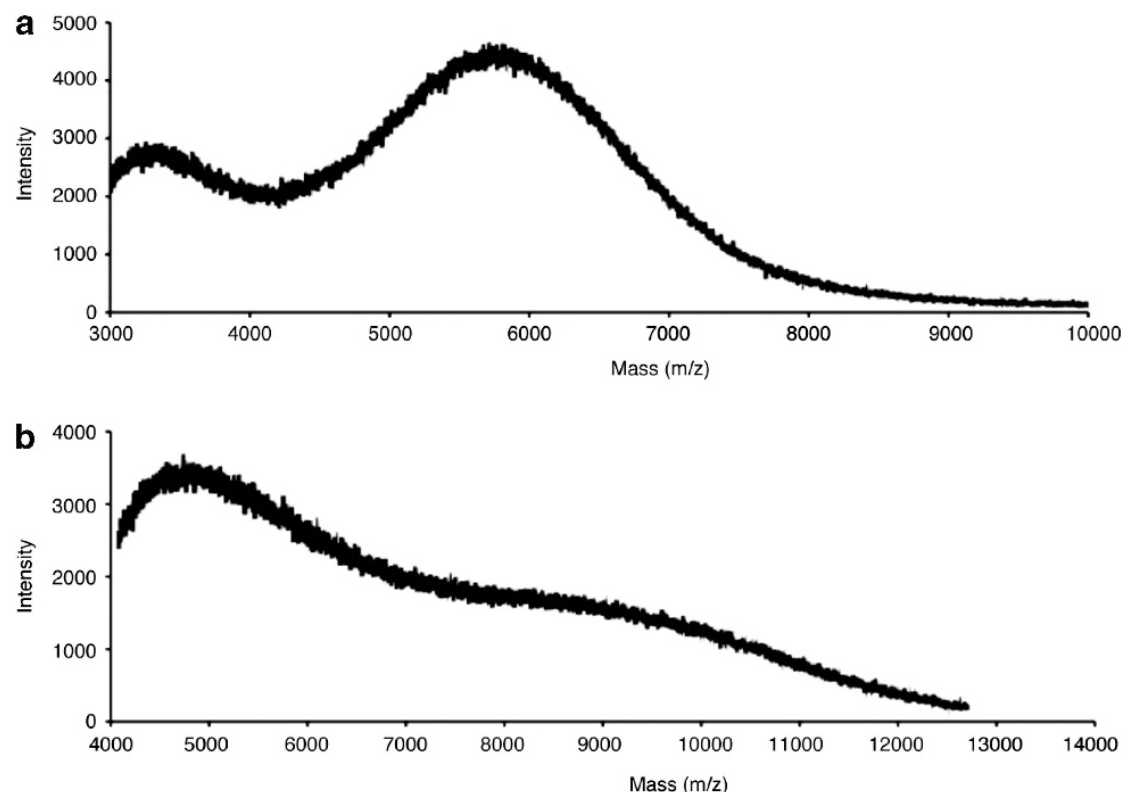

Figure 1 MALDI-TOF-MS spectra for (a) POSS-PIm16Br and (b) POSS-PIm32Br. 

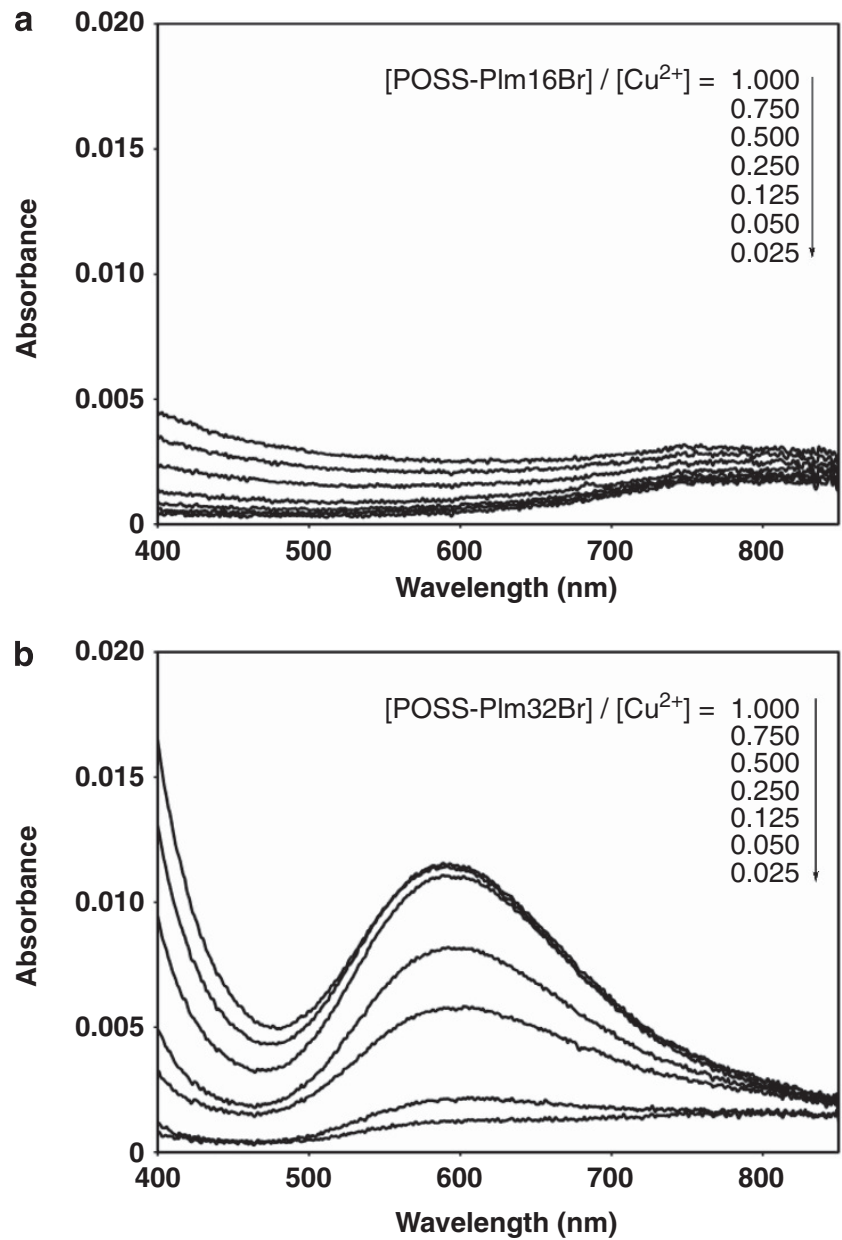

Figure 3 Spectrophotometric titrations of (a) POSS-PIm16Br and (b) POSS$\mathrm{PIm} 32 \mathrm{Br}$ against $\mathrm{Cu}^{2+}$ ions. The number of $\mathrm{Cu}^{2+}$ ions in the solutions was held constant at $0.1 \mathrm{~mm}$.

dendrimer previously reported, a $\mathrm{Cu}^{2+}-\mathrm{N}_{2} \mathrm{O}_{2}$ complex can be formed. ${ }^{20}$ For all concentrations of $\mathrm{Cu}^{2+}$ ions and POSS-PIm32Br, the absorption maxima values were equal at $600 \mathrm{~nm}$. The absorption intensity increased with increasing the concentration ratio of POSSPIm32Br to $\mathrm{Cu}^{2+}$ ions, but no further increases were observed beyond a ratio of 0.5 . The data indicate that the $\mathrm{Cu}^{2+}$-binding capacity of POSS-PIm32Br was approximately two $\mathrm{Cu}^{2+}$ ions. Although 24 tertiary amines in the branches of POSS-PIm32Br potentially coordinate with $\mathrm{Cu}^{2+}$ ions, the present data suggest that few amine units in POSS-PIm32Br were used to form $\mathrm{Cu}^{2+}{ }_{-} \mathrm{N}_{4}$ complexes due to depression of the movement of the terminal groups.

Synthesis and characterization of imidazolium $\mathrm{PF}_{6}$-terminated POSS-Core dendrimers

As 1-butyl-3-methylimidazolium hexafluorophosphate $\left(\mathrm{PF}_{6}{ }^{-}\right)$is historically the most studied room temperature ionic liquid, imidazolium $\mathrm{PF}_{6}$-terminated POSS-core dendrimers were prepared by anion exchange reactions of POSS-PIm16Br and POSS-PIm32Br to estimate their assembled bulk nanostructures. The dendrimer, POSSPIm16Br or POSS-PIm32Br, was dissolved in a minimal volume of water. After $\mathrm{KPF}_{6}$ aqueous solution was added to the aqueous dendrimer solution, white precipitates were observed. The
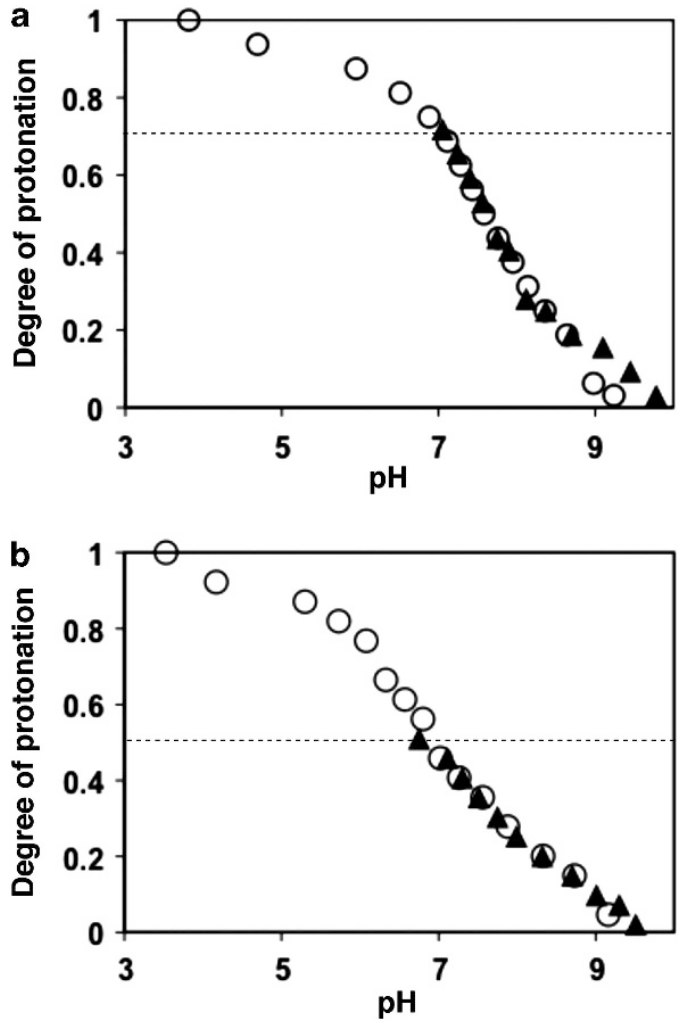

Figure 4 Potentiometric titrations of (a) POSS-PIm16PF6 and (b) POSSPIm32PF6 in $2.0 \mathrm{~m} \mathrm{NaCl}$ solution with $0.1 \mathrm{~m} \mathrm{NaOH}$ solution. The initial $\mathrm{pH}$ titrations of the as-prepared dendrimer solutions $(\boldsymbol{\Lambda})$ and those of the acidified aqueous solutions $(O)$ are both shown. [POSS-PIm16PF6] $=0.01 \mathrm{mmol}$. [POSS-PIm32PF6] $=0.004 \mathrm{mmol}$.

precipitated products were isolated by decantation, washed with water and dried under vacuum. The resulting crude products were washed several times with pure water until no residual bromide anion was detected with the use of $\mathrm{AgNO}_{3}$ and imidazolium $\mathrm{PF}_{6}$-terminated first and second generation POSS-core dendrimers (POSS-PIm16 $\mathbf{P F}_{6}$ and POSS-PIm32 PF $_{6}$, respectively) were obtained. Both dendrimers were soluble in DMF, DMSO and acetone; partially soluble in methanol; and insoluble in $\mathrm{H}_{2} \mathrm{O}$ and chloroform. The elemental analysis of POSS-PIm16 $\mathbf{P F}_{6}$ matched that of a fully functionalized dendrimer with $19.3 \mathrm{PF}_{6}$ that is associated with $5 \mathrm{H}_{2} \mathrm{O}$ molecules per dendrimer. The elemental analysis of POSS-Im32PF 6 matched that of a dendrimer missing four branches with $35 \mathrm{PF}_{6}$ that is associated with $6 \mathrm{H}_{2} \mathrm{O}$ molecules per dendrimer. These $\mathrm{PF}_{6}$ contents are higher than those of the terminal imidazolium cations for POSS-PIm16 $\mathbf{P F}_{6}$ and POSS-PIm32 PF $_{6}$.

The following experiments were conducted to estimate the degrees of protonation of the branched tertiary amines of the obtained dendrimers. The obtained dendrimers were dissolved in $2 \mathrm{M} \mathrm{NaCl}$ aqueous solution containing $20 \mathrm{vol} \%$ methanol. The $\mathrm{pH}$ titrations of the dendrimer solutions were first performed with $0.1 \mathrm{M} \mathrm{NaOH}$ aqueous solution. The basic solutions from the first $\mathrm{pH}$ titrations were then acidified to $\mathrm{pH} 2$ by $0.25 \mathrm{M} \mathrm{HCl}$ aqueous solution, and $\mathrm{pH}$ titrations were again performed with $0.1 \mathrm{M} \mathrm{NaOH}$ aqueous solution. Figure 4 shows that the degrees of protonation of the branched tertiary amines of POSS-PIm16 PF $_{6}$ and POSS-PIm32 PF 6 were 0.70 and 0.50 , respectively. The numbers of anion exchanging sites for the tertiary amine groups in POSS-PIm16 $\mathbf{P F}_{6}$ and POSS- 
PIm32PF 6 were thus calculated as 5.6 and 12 , respectively. Therefore, the total numbers of anion exchanging sites in POSS-PIm16 $\mathbf{P F}_{6}$ and POSS-PIm32 PF $_{6}$, including both the tertiary amines and imidazolium cations, were 21.6 and 44, respectively. These values are in fairly good agreement with the estimated values from elemental analysis.

${ }^{1} \mathrm{H}-\mathrm{NMR}$ analysis showed that the degree of terminal functionalization in POSS-PIm16 $\mathbf{P F}_{6}$ was $96 \%$, obtained by calculating and comparing the areas of the peaks from the aromatic protons present on the imidazolium ring and the methyl protons on the $n$-propyl groups with the integrations of peaks from methylene protons on the POSS-core. The ${ }^{1} \mathrm{H}-\mathrm{NMR}$ analysis for POSS-PIm32PF $\mathbf{F}_{6}$, however, showed that integrations for the protons on the dendrons compared with integrations from the methylene protons on the POSS-core showed $45 \%$ of the theoretical fully functionalized POSS-PIm32PF 6 . As elemental analysis supported the structure, and the starting dendrimer, POSS-PIm32Br, was fully characterized, the reduction in the integration corresponding to the terminal units may be due to longer relaxation times for those groups than those for the methylene protons on the POSS-core. The integration ratios for the aromatic protons present on the imidazolium ring and the methyl protons on the $n$-propyl groups against the theoretical fully functionalized POSSPIm32 $\mathbf{P F}_{6}$ increased from 45 to $60 \%$ when the recycle delay (d1) was increased from 1 to $20 \mathrm{~s}$ in the ${ }^{1} \mathrm{H}-\mathrm{NMR}$ analysis.

Thermal and WAXS studies of Imidazolium $\mathrm{PF}_{6}$-terminated POSScore dendrimers

When acetone solutions of POSS-PIm16 $\mathbf{P F}_{6}$ and POSS-PIm32 $3 \mathbf{P F}_{6}$ were cast on a Teflon sheet and heated at $60{ }^{\circ} \mathrm{C}$, optically transparent films were obtained. Although the films hardened upon cooling to $0{ }^{\circ} \mathrm{C}$, they softened and formed sticky gums at moderate temperature. As we used the modulated DSC apparatus, the effects of unknown irreversible processes on the apparent DSC curve could be eliminated, which enabled us measurement of the heat flow due to a thermoreversible process such as glass transition or melting. The obtained thermo-reversible heat flow is plotted in Figure 5. For both samples, the glass transition was clearly observed in the temperature range shown here. Although the mid-point temperature is almost the same, a wider glass transition temperature range was observed for POSS-PIm16 PF $_{6}$. This result indicates a wider distribution in the mobility of a segment undergoing the glass transition, which in turn suggests that there is a wider distribution of physical constraints in POSS-PIm16 PF $_{6}$. This result further implies that the physical constraints can be homogenized as an increase in the generation number of the dendrimer. The transition temperature range here is notably wider than that of an ordinary polymer, even that of POSS-PIm32 $\mathrm{PF}_{6}$, indicating inhomogeneity of physical constraints because of the characteristic molecular structure of the dendrimer. There is also a quite noticeable endothermic peak near the end-point of the glass transition of both samples, which is reminiscent of hysteresis peak due to physical aging (enthalpy relaxation); however, this is not the case because the DSC curves presented here are thermo-reversible heat flows measured using the modulated DSC apparatus. Although the reason for the endothermic peak near the end-point of the glass transition is unknown at present, we speculate that partial protonation of the branched tertiary amine units might cause such a complex DSC thermogram. We are now preparing imidazolium-terminated POSS-core dendrimers with non-ionic branches for more detailed study of their thermal behaviors.

To determine the assembly motif of the POSS-core dendrimers, WAXS measurements were made of the cast films of POSS-PIm16 $\mathbf{P F}_{6}$ and POSS-PIm32PF 6 . Both sets of WAXS data are characterized by two amorphous halos: a strong peak around $q=15 \mathrm{~nm}^{-1}$ and another tiny peak around $q=10 \mathrm{~nm}^{-1}$ (Figure 6). These features are related to structural correlations. According to a previous report, wide-angle neutron scattering (WANS) and WAXS data for 1-butyl-3methylimidazolium hexafluorophosphate $\left([\mathrm{bmin}]\left[\mathrm{PF}_{6}\right]\right.$ ) show one strong peak at $q=15 \mathrm{~nm}^{-1}$ and another at $q=10 \mathrm{~nm}^{-1}$. $^{34}$ These spatial correlations were previously considered to be evidence of residual order at a microscopic level in the liquid $[\mathrm{bmin}]\left[\mathrm{PF}_{6}\right] .{ }^{35}$ Recent simulation studies suggest that the broad peak at $q=15 \mathrm{~nm}^{-1}$ is due to intramolecular and close contact intermolecular interactions, ${ }^{36}$ and the characteristic distance of $\sim 0.63 \mathrm{~nm}$ corresponding to the peak at $q=10 \mathrm{~nm}^{-1}$ is related to the first

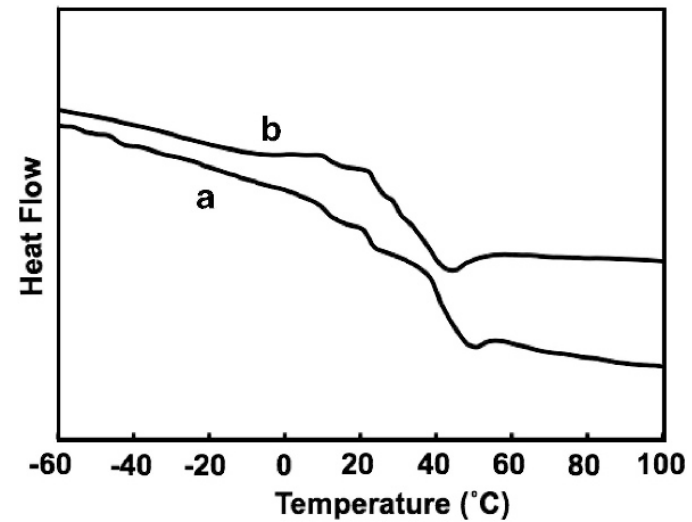

Figure 5 Differential scanning calorimetry (DSC) traces for (a) POSSPIm16PF 6 and (b) POSS-PIm32 $\mathrm{PF}_{6}$ at a heating rate of $10{ }^{\circ} \mathrm{Cmin}^{-1}$ in $\mathrm{N}_{2}$ flow over the entire temperature range (3rd run).

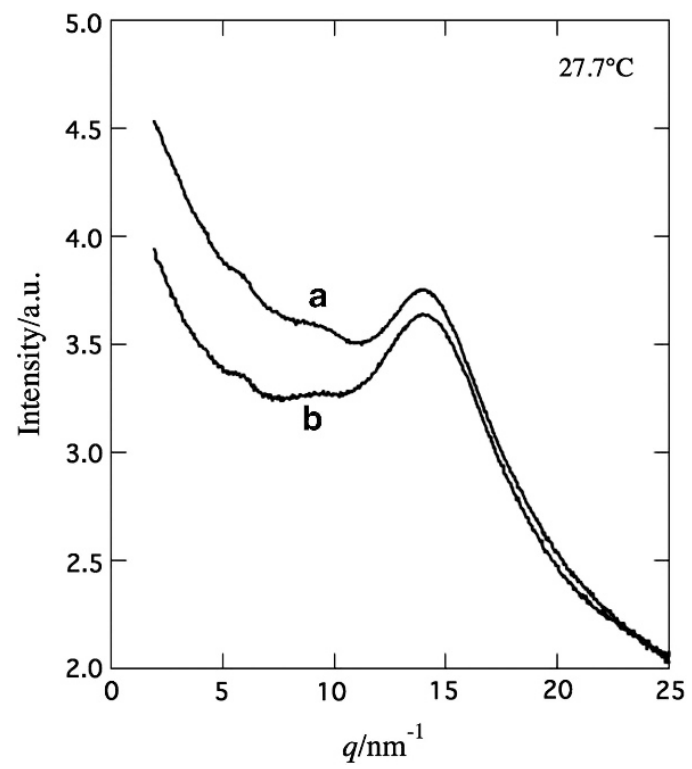

Figure 6 Wide-angle X-ray scattering (WAXS) data for cast films of (a) POSS-PIm16PF 6 and (b) POSS-PIm32 PF $_{6}$ at $27.7{ }^{\circ} \mathrm{C}$. 
solvation shell of the $\mathrm{PF}_{6}$ anion around the imidazolium ring. ${ }^{37-39}$ Our X-ray diffraction data for the cast films of POSS-PIm16PF 6 and POSS-PIm32PF 6 can be considered to reflect the same features that were described by the WANS data for $[\mathrm{bmin}]\left[\mathrm{PF}_{6}\right] .{ }^{35}$ Our observations suggest that domains similar to dialkyl imidazolium hexafluorophosphate formed in the cast films of POSS-PIm16 $\mathbf{P F}_{6}$ and POSS-PIm32PF 6 .

WAXS data were also collected for both samples over a temperature range of -170 to $100^{\circ} \mathrm{C}$. The strong peaks around $q=15 \mathrm{~nm}^{-1}$ of both dendrimers shifted to smaller $q$ values with increasing temperature (see Supplementary Information). The spacing evaluated from the peak position $\left(q^{*}\right)$ was plotted as a function of the temperature; $d$ was evaluated as $d=2 \pi / q^{*}$. The plots for both dendrimers exhibit a similar sharp change of slope (Figure 7). The critical temperatures (inflection points) for POSS-PIm16 $\mathbf{P F}_{6}$ and POSS-PIm32 $\mathbf{P F}_{6}$ are 4 and $11^{\circ} \mathrm{C}$, respectively. The previous WANS data for $[\mathrm{bmin}]\left[\mathrm{PF}_{6}\right]$ also show a similar temperature dependence, with an inflection at $-73{ }^{\circ} \mathrm{C}$ that correspond to its $T_{\mathrm{g}}$ as determined by heat capacity measurements. ${ }^{35}$ This results implies that the inflection of the temperature dependence of $d$ (spacing) can be correlated to the glass transition. Our WAXS data show an inflection temperature close to the onset temperature of the glass transition in the DSC curves. This finding suggests that domains similar to dialkyl imidazolium hexafluorophosphate exist in the cast films of POSS-PIm16 $\mathbf{P F}_{6}$ and POSS-PIm32PF 6 .

Ionic conductivity of imidazolium TFSI-terminated POSS-Core dendrimers

Imidazolium TFSI-terminated POSS-core dendrimers were prepared by an anion exchange reaction for ionic conductivity measurements. After the dendrimer, POSS-PImBr16 or POSS-PImBr32, was dissolved in water, an aqueous solution of LiTFSI (1.5 equivalent of the imidazolium units) was added to the dendrimer aqueous solution. The precipitated products were isolated by decantation, washed with water and dried under vacuum to obtain imidazolium TFSI-terminated first and second generation POSS-core dendrimers, denoted as POSS-PIm16TFSI and POSS-PIm32TFSI, respectively. The elemental analysis of POSS-PIm16TFSI matched that of a fully functionalized dendrimer with 19 TFSI that is associated with $10 \mathrm{H}_{2} \mathrm{O}$ molecules per

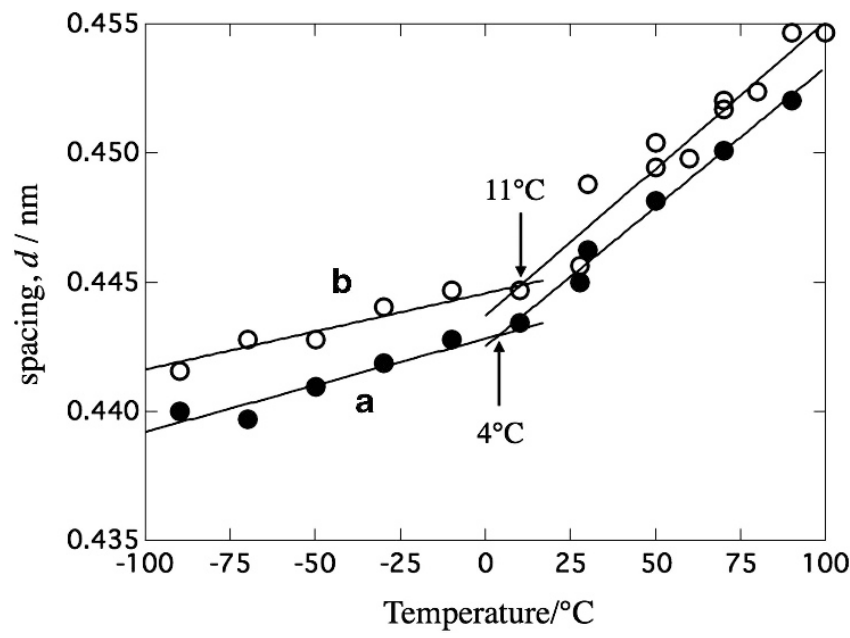

Figure 7 Temperature dependence of spacing, $d$, as evaluated from the peak position in the wide-angle X-ray scattering (WAXS) profile for (a) POSS-PIm16PF 6 and (b) POSS-PIm32PF dendrimer. The elemental analysis of POSS-PIm32TFSI matched that of a dendrimer missing four branches with 35.5 TFSI that is associated with $15 \mathrm{H}_{2} \mathrm{O}$ molecules per dendrimer. These TFSI contents are higher than those of the terminal imidazolium cations in POSS-PIm16TFSI and POSS-PIm32TFSI, similar to the case of POSS-PIm16 PF $_{6}$ and POSS-PIm32 PF $_{6}$. The following experiments were performed to estimate the degrees of protonation of the branched tertiary amines of the obtained dendrimers. The dendrimers were dissolved in a $2-\mathrm{M} \mathrm{NaCl}$ aqueous solution containing $15 \mathrm{vol} \%$ methanol. The $\mathrm{pH}$ titrations for the dendrimer solutions were first performed with $0.1 \mathrm{M} \mathrm{NaOH}$ aqueous solution. The basic solutions from the first $\mathrm{pH}$ titrations were then acidified to $\mathrm{pH} 2$ by $0.25 \mathrm{M} \mathrm{HCl}$ aqueous solution, and $\mathrm{pH}$ titrations were again performed with $0.1 \mathrm{M}$ $\mathrm{NaOH}$ aqueous solution. These results suggested that the degrees of protonation of the branched tertiary amine groups in POSSPIm16TFSI and POSS-PIm32TFSI were 0.54 and 0.39, respectively (Figure 4). The numbers of anion exchanging sites in the tertiary amine groups in POSS-PIm16TFSI and POSS-PIm32TFSI were thus calculated as 4.3 and 9.4, respectively. Therefore, the total numbers of anion exchanging sites in POSS-PIm16TFSI and POSS-PIm32TFSI, including both tertiary amines and imidazolium cations, were 20 and 41 , respectively. These values are in fairly good agreement with the estimated values from elemental analysis.

The thermal stability of the dendrimers containing LiTFSI was verified by TGA. POSS-PIm16TFSI and POSS-PIm16TFSI containing 0.1 equivalent LiTFSI showed 5 wt $\%$ losses at 307 and $305^{\circ} \mathrm{C}$, respectively. DSC thermograms of POSS-PIm16TFSI and POSS-PIm32TFSI showed relatively intense glass transitions at -22 and $-23{ }^{\circ} \mathrm{C}$, respectively, which are lower than those of POSSPIm16PF $_{6}$ and POSS-PIm32PF 6 (see Supplementary Information). Generally, the addition of LiTFSI to ionic liquids induces an increase in $T_{\mathrm{g}}$, which may be due to specific interaction between LiTFSI and the ionic liquids. The glass transition temperatures of POSSPIm16TFSI and POSS-PIm32TFSI, however, decreased with LiTFSI addition (Table 1). In all DSC thermograms, $T_{\mathrm{g}}$-like weak baseline shifts were also observed around $20^{\circ} \mathrm{C}$ (see Supplementary Information). The presence of two $T_{\mathrm{g}}$ is tentatively explained by distinct nanophase separation between two parts of the dendrimer assemblies.

The ionic conductivities of POSS-PIm16TFSI and POSSPIm32TFSI containing LiTFSI salts were estimated by the ac impedance method after drying the samples thoroughly. The conductivity evolution with temperature of POSS-PIm16TFSI and POSS-PIm32TFSI, containing different concentrations of LiTFSI, is displayed in Figure 8. The obtained samples had ionic conductivities between $10^{-6}$ and $10^{-5} \mathrm{~S} \mathrm{~cm}^{-1}$, comparable to those of typical polymeric electrolytes. ${ }^{40-42}$ A maximum ionic conductivity

Table $1 T_{\mathrm{g}}$ and ionic conductivity of the POSS-core dendrimers

\begin{tabular}{lcccc}
\hline Sample & Dendrimer & $\begin{array}{c}{\left[\mathrm{Li}^{+}\right] /} \\
\text {[imidazolium unit] }\end{array}$ & $\begin{array}{c}\mathrm{T}_{g}\left({ }^{\circ} \mathrm{C}\right) \\
\text { lonic } \\
\text { conductivity }\end{array}$ \\
\hline 1 & POSS-PIm16TFSI & 0.1 & -29 & $4.0 \times 10^{-5}$ \\
2 & POSS-PIm16TFSI & 0.3 & -29 & $3.2 \times 10^{-5}$ \\
3 & POSS-PIm16TFSI & 0.5 & -37 & $2.5 \times 10^{-5}$ \\
4 & POSS-PIm32TFSI & 0.1 & -35 & $2.3 \times 10^{-5}$ \\
5 & POSS-PIm32TFSI & 0.5 & -36 & $9.3 \times 10^{-6}$ \\
\hline
\end{tabular}

Abbreviation: POSS, polyhedral oligomeric silsesquioxane.

${ }^{a}$ At $51^{\circ} \mathrm{C}$. Measured by ac impedance method. 


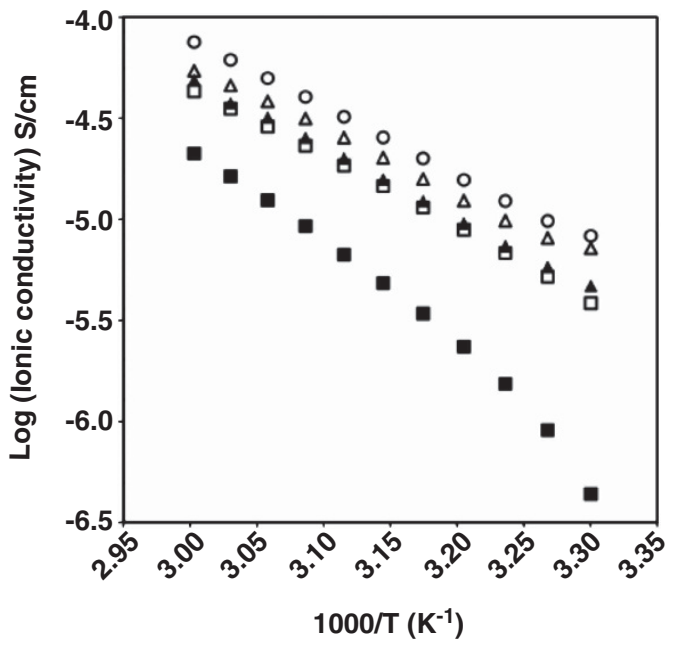

Figure 8 Temperature dependence of ionic conductivity of POSSPIm16TFSI and POSS-PIm32TFSI containing different concentration of LiTFSI. Samples $1(\bigcirc), 2(\triangle), 3(\mathbf{\Delta}), 4(\square)$ and $5(\mathbf{\square})$ are defined in Table 1.

Table 2 VFT parameters of the POSS-core dendrimers

\begin{tabular}{|c|c|c|c|c|c|}
\hline Sample & & $\begin{array}{l}\mathrm{T}_{0} \\
(K)\end{array}$ & $\begin{array}{c}\mathrm{A} \\
\left(\mathrm{Scm}^{-1} \mathrm{~K}^{1 / 2}\right)\end{array}$ & $\begin{array}{l}\mathrm{B} \\
(K)\end{array}$ & $R M S$ \\
\hline $\begin{array}{l}1 \\
2 \\
3 \\
5\end{array}$ & $\begin{array}{l}\text { POSS-PIm 16TFSI: LiTFSI }=1: 0.1 \\
\text { POSS-PIm 16TFSI: LiTFSI }=1: 0.3 \\
\text { POSS-PIm 16TFSI: LiTFSI }=1: 0.5 \\
\text { POSS-PIm32TFSI: LiTFSI }=1: 0.5\end{array}$ & $\begin{array}{l}150 \\
150 \\
150 \\
200\end{array}$ & $\begin{array}{r}254.9 \\
108.0 \\
247.2 \\
76.5\end{array}$ & $\begin{array}{l}2222 \\
2119 \\
2298 \\
1602\end{array}$ & $\begin{array}{l}0.9995 \\
0.9989 \\
0.9993 \\
0.9997\end{array}$ \\
\hline
\end{tabular}

Abbreviations: POSS, polyhedral oligomeric silsesquioxane; VFT, Vogel-Fulcher-Tamman.

of $4.03 \times 10^{-5}$ at $50{ }^{\circ} \mathrm{C}$ was observed for the POSS-core dendrimer of lower generation and less lithium salt addition. The lower conductivities of the higher generation dendrimer and those with greater lithium salt additions may be due to increased viscosity or may have resulted from specific interactions involving the formation of ionic clusters.

Vogel-Fulcher-Tamman (VFT) plots were also fitted to obtain further information on the ionic conductivities of the samples. ${ }^{43-45}$ Generally, the temperature dependence of the ionic conductivity of a polymer electrolyte can be adequately described for amorphous polymer electrolytes by the VFT equation, $\sigma(T)=A T^{-1 / 2} \exp$ $\left[-B /\left(T-T_{0}\right)\right]$, where $\sigma$ and $T$ are the ionic conductivity and absolute temperature, respectively. Terms $A, B$ and $T_{0}$ are fitting parameters that correspond to the carrier ion number, pseudoactivation energy associated with the motion of the polymer segment, and quasi-equilibrium glass transition temperature of the electrolyte, respectively. VFT parameters obtained by fitting the VFT equation to the experimental conductivity data are summarized in Table 2. This VFT-type behavior indicates that segmental motion of the present dendrimer electrolytes may contibute to long-range charge transport, as in polymer electrolytes composed of oligo(ethyleneoxide) chains. The ionic conductivity of POSS-PIm32TFSI containing $0.5 \mathrm{~mol} \%$ of LiTFSI was significantly lower than that of POSSPIm16TFSI. The lower ionic conductivity is due to the significantly lower dissociation degree of the ionic species for the former sample, as shown by the smaller value of VFT parameter A, corresponding to the carrier ion number, despite the lower activation energy of the former sample, corresponding to the lower value of VFT parameter B. The lower value of parameter B for POSS-PIm32TFSI suggests higher flexibility related to the mobility of lithium cations, which implies that an increase in generation number reduces the activation energy for ion transport derived from a decrease in the viscosity of the ion transport path from the shorter distance between terminal ion hopping sites. However, the dissociation degree of the ionic species decreased with increasing generation number. Although increasing LiTFSI content of POSS-PIm16TFSI from 0.1 to $0.3 \mathrm{~mol} \%$ for decreased the activation energy, the carrier ion number decreased, suggesting that the lower ionic conductivity for sample 2 (defined in Table 1) is attributed to the lower dissociation degree of the ionic species. These data also suggest that the improved ionic conductivity of sample 1 is attributed to the high dissociation degree of the ionic species.

\section{CONCLUSIONS}

We synthesized imidazolium salt-terminated POSS-core PAMAMtyped dendrimers and studied their solution, bulk properties and polyelectrolyte function. The structures of the dendrimers were identified on the basis of multinuclear NMR data and elemental analysis. The results from $\mathrm{pH}$ titration and coordination with $\mathrm{Cu}^{2+}$ ions of the imidazolium bromide-terminated first and second generation PAMAM-typed POSS-core dendrimers (POSS-PIm16Br and POSS-PIm32Br, respectively) in aqueous solutions indicated that the ammonium groups in POSS-PIm16Br were not significantly more deprotonated or coordinated with $\mathrm{Cu}^{2}+$ ions than those in POSS-PIm32Br. In the case of POSS-PIm32Br, the deprotonation of the 16 amine groups in the second shell was easier to achieve than that of the 8 amine groups in the first shell, and few amine units were used to form $\mathrm{Cu}^{2+}-\mathrm{N}_{4}$-type complexes due to depression of the movement of the terminal groups. WAXS data for cast films of the imidazolium $\mathrm{PF}_{6}$-terminated first and second generation POSScore dendrimers, which were prepared by anion exchange reactions of POSS-PIm16Br and POSS-PIm32Br, were characterized by two amorphous halos, with a strong peak around $q=15 \mathrm{~nm}^{-1}$ and another tiny peak around $q=10 \mathrm{~nm}^{-1}$. We also found that the strong peaks around $q=15 \mathrm{~nm}^{-1}$ for both dendrimers showed two temperature dependences, and the observed inflection temperatures for POSS-PIm16PF 6 and POSS-PIm32PF 6 were 4 and $11^{\circ} \mathrm{C}$, respectively, corresponding to the onset temperatures of the glass transition. Our X-ray diffraction data for the cast films of POSS-PIm16 PF $_{6}$ and POSS-PIm32PF 6 revealed the same features that were described for 1-butyl-3-methylimidazolium hexafluorophosphate. These observations suggest that domains similar to dialkyl imidazolium hexafluorophosphate formed in the cast films of POSS-PIm16 PF $_{6}$ and POSS-PIm32 PF 6 . The ionic conductivities of POSS-PIm16TFSI and POSS-PIm32TFSI containing LiTFSI salts were estimated by the ac impedance method. A maximum ionic conductivity of $4.03 \times 10^{-5}$ at $51^{\circ} \mathrm{C}$ was observed for the POSS-core dendrimer of lower generation and less lithium salt addition. The VFT-type behavior indicates that segmental motion of the these dendrimer electrolytes may contribute to long-range charge transport, as in polymer electrolytes composed of oligo(ethyleneoxide) chains. This report gives the first example of POSS-core dendrimers terminated with ionic liquids, which are ideal candidates for the construction of ordered nano-sized network architectures for solid ionic conductive materials. We are now optimizing branched and terminal structures to increase ionic conductivity. 


\section{ACKNOWLEDGEMENTS}

This study is a part of the Kyoto City Collaboration of Regional Entities for the Advancement of Technology Excellence of JST, Grant-in-Aid for Scientific Research (no. 24350058) from the Ministry of Education, Culture, Sports Science and Technology, Government of Japan, and a Grant-in-Aid for Scientific Research on Innovative Areas 'New Polymeric Materials Based on Element-Blocks (no. 2401)' (24102003) of The Ministry of Education, Culture, Sports, Science and Technology, Japan. We thank Professor Tsuyoshi Kawai of Nara Institute of Science and Technology for MALDI-TOF-MS measurements, supported by Kyoto-Advance Nanotechnology Network, and Professor Katsuhiro Yamamoto for utilization of Linkam LK-600 M. We are also indebted to Dr Takahiro Kusukawa (Department of Chemical and Materials Technology, Graduate School of Science and Technology, Kyoto Institute of Technology) for generous help with the NMR measurements.

1 Welton, T. Room-temperature ionic liquids. Solvents for synthesis and catalysis. Chem. Rev. 99, 2071-2084 (1999).

2 Ueki, T. \& Watanabe, M. Macromolecules in ionic liquids: progress, challenges, and opportunities. Macromolecules 41, 3739-3749 (2008).

3 Ohno, H. Functional design of ionic liquids. Bull. Chem. Soc. Jpn. 79, 1665-1680 (2006).

4 Kubo, W., Murakoshi, K., Kitamura, T., Wada, Y., Hanabusa, K., Shirai, H. \& Yanagida, S. Fabrication of quasi-solid-state dye-sensitized $\mathrm{TiO} 2$ solar cells using Low molecular weight gelators. Chem. Lett. 1241-1242 (1998).

5 Kubo, W., Kambe, S., Nakade, S., Kitamura, T., Hanabusa, K., Wada, Y. \& Yanagida, S Photocurrent-determining processes in quasi-solid-state dye-sensitized solar cells using ionic gel electrolytes. J. Phys. Chem. B 107, 4374-4381 (2003)

6 Ikeda, A., Sonoda, K., Ayabe, M., Tamaru, S., Nakashima, T., Kimizuka, N. \& Shinkai, S. Gelation of ionic liquids with a low molecular-weight gelator showing T-gel above $100^{\circ} \mathrm{C}$. Chem. Lett. 1154-1155 (2001).

7 Neouze, M. A., Le Bideau, J., Leroux, F. \& Vioux, A. A route to heat resistant solid membranes with performances of liquid electrolytes. Chem. Commun. 41, 1082-1084 (2005)

8 Shi, F., Fang, Q., Li, D. \& Deng, Y. Silica-gel-confined ionic liquids: a new attempt for the development of supported nanoliquid catalysis. Chem. Eur. J. 11, 5279-5288 (2005).

9 Green, O., Grubjesic, S., Lee, S. \& Firestone, M. The design of polymeric ionic liquids for the preparation of functional materials. Polym. Rev. 49, 339-360 (2009).

10 Armand, M., Endres, F., MacFarlane, D. R., Ohno, H. \& Scrosati, B. Ionic-liquid materials for the electrochemical challenges of the future. Nat. Mater. 8, 621-629 (2009).

11 Carlin, R. T. \& Fuller, J. Ionic liquid-polymer gel catalytic membrane. Chem. Commun. 33, 1345-1346 (1997).

12 Tang, J., Sun, W., Tang, H., Radosz, M. \& Shen, Y. Enhanced CO2 absorption of poly(ionic liquid)s. Macromolecules 38, 2037-2039 (2005).

13 Tang, J., Tang, H., Sun, W., Plancher, H., Radosz, M. \& Shen, Y. Poly(ionic liquid)s: a new material with enhanced and fast $\mathrm{CO}_{2}$ absorption. Chem. Commun. 41 3325-3327 (2005).

14 Tang, J., Radosz, M. \& Shen, Y. Poly(ionic liquid)s as optically transparent microwaveabsorbing materials. Macromolecules 41, 493-496 (2008).

15 Weber, R. L., Ye, Y., Schmitt, A. L., Banik, S. M., Elabd, Y. A. \& Mahanthappa, M. K. Effect of nanoscale morphology on the conductivity of polymerized ionic liquid block copolymers. Macromolecules 44, 5727-5735 (2011)

16 Wiesenauer, E. F., Edwards, J. P., Scalfani, V. F., Bailey, T. S. \& Gin, D. L. Synthesis and ordered phase separation of imidazolium-based alkyl-ionic diblock copolymers made via ROMP. Macromolecules 44, 5075-5078 (2011).

17 Jaffrés, P. -A. \& Morris, R. E. Synthesis of highly functionalised dendrimers based on polyhedral silsesquioxane cores. J. Chem. Soc. Dalton Trans. 27, 2767-2770 (1998).

18 Zhang, X., Haxton, K. J., Ropartz, L., Cole-Hamilton, D. J. \& Morris, R. E. Synthesis and computer modelling of hydroxy-derivatised carbosilane dendrimers based on polyhedral silsesquioxane cores. J. Chem. Soc. Dalton Trans. 30, 3261-3268 (2001).

19 Haxton, K. J., Cole-Hamilton, D. J. \& Morris, R. E. Silsesquioxane dendrimers as catalysts: a Bite-sized Molecular Dynamics Study. Dalton Trans. 36, 3415-3420 (2007).

20 Naka, K., Fujita, M., Tanaka, K. \& Chujo, Y. Water-soluble anionic POSS-Core dendrimer: synthesis and copper(II) complexes in aqueous solution. Langmuir 23, 9057-9063 (2007)
21 Tanaka, K., Inafuku, K., Naka, K. \& Chujo, Y. Enhancement of entrapping ability of dendrimers by a cubic silsesquioxane core. Org. Biomol. Chem. 6, 3899-3901 (2008).

22 Naka, K., Masuoka, S., Shinke, R. \& Yamada, M. Synthesis of first- and secondgeneration imidazole-terminated POSS-core dendrimers and their $\mathrm{pH}$ responsive and coordination properties. Polym. J. 44, 353-359 (2012).

23 Tanaka, K., Ishiguro, F. \& Chujo, Y. POSS Ionic Liquid. J. Am. Chem. Soc. 132, 17649-17651 (2010)

24 Tanaka, K., Ishiguro, F. \& Chujo, Y. Thermodynamic Study of POSS-based ionic liquids with various numbers of ion pairs. Polym. J. 43, 708-713 (2011).

25 Huang, J. -F., Luo, H., Liang, C., Sun, I. -W., Baker, G. A. \& Dai, S. Hydrophobic Brörnsted acid-base ionic liquids based on PAMAM dendrimers with high proton conductivity and blue photoluminescence. J. Am. Chem. Soc. 127, 12784-12785 (2005).

26 Chen, C. \& Fang, X. Synthesis and conductivity performance of hyperbranched polymer electrolytes with terminal ionic groups. J. Appl. Polym. Sci. 117, 35393544 (2010).

27 Müller, R., Laschober, C., Szymanski, W. W. \& Allmaier, G. Determination of Molecular weight, particle size, and density of high number generation PAMAM dendrimers using MALDI-TOF-MS and nES-GEMMA. Mancromolecules 40, 5599-5605 (2007).

28 Ojima, H. \& Sone, K. Absorption spectra and catalytic behavior of the copper(II) chelates of some alkylated ethylenediamines. Bull. Chem. Soc. Jpn 35, 298-303 (1962).

29 Jonassen, H. B. \& Dexter, T. H. Inorganic Complex Compounds Containing Polydentate Groups. I. The complex ions formed Between copper(II) ions and ethylenediamine. J. Am. Chem. Soc. 71, 1553-1556 (1949).

30 Edsall, J. D., Felsenfeld, G., Goodman, D. S. \& Gurd, F. R. N. The association of imidazole with the ions of zinc and cupric copper. J. Am. Chem. Soc. 76, 3054-3061 (1954).

31 Jonassen, H. B., Reeves, R. E. \& Segal, L. Inorganic Complex Compounds Containing Polydentate Groups. XI. Effect of hydroxide Ion on the bis-ethylenediaminecopper(II) ion. J. Am. Chem. Soc. 77, 2748-2749 (1955).

32 Billo, E. J. Copper(II) chromosomes and the rule of average environment. Inorg. Nucl. Chem. Lett. 10, 613-617 (1974).

33 Krot, K. A., de Namor, A. F. D., Aguilar-Cornejo, A. \& Nolan, K. B. Speciation, stability constants and structures of complexes of copper(II), nickel(II), silver(I) and mercury(II) with PAMAM dendrimer and related tetraamide ligands. Inorg. Chim. Acta 358, 3497-3505 (2005).

34 Triolo, A., Mandanici, A., Russina, O., Rodriguez-Mora, V., Cutroni, M., Hardacre, C., Nieuwenhuyzen, M., Bleif, H. -J., Keller, L. \& Ramos, M. A. Thermodynamics, structure, and dynamics in room temperature ionic liquids: the case of 1-butyl-3methyl imidazolium hexafluorophosphate ([bmim][PF6]). J. Phys. Chem. B 110, 21357-21364 (2006)

35 Dibrov, S. M. \& Kochi, J. K. Crystallographic view of fluidic structures for room-temperature ionic liquids: 1-butyl-3-methylimidazolium hexafluorophosphate. Acta Crystallogr. C 62, o19-021 (2006).

36 Annapureddy, H. V. R., Kashyap, H. K., De Biase, P. M. \& Margulis, C. J. What is the Origin of the prepeak in the $\mathrm{X}$-ray scattering of imidazolium-based room-temperature ionic liquids? J. Phys. Chem B 114, 16838-16846 (2010).

37 Morrow, T. I. \& Maginn, E. J. Molecular dynamics study of the ionic liquid 1-n-butyl-3methylimidazolium hexafluorophosphate. J. Phys. Chem. B 106, 12807-12813 (2002).

38 Urahata, S. M. \& Ribeiro, M. C. C. Structure of ionic liquids of 1-alkyl-3-methylimidazolium cations: a Systematic Computer Simulation Study. J. Chem. Phys. 120, 1855-1863 (2004).

39 Hardacre, C., McMath, S. E. J., Nieuwenhuyzen, M., Bowron, D. T. \& Soper, A. K. Liquid structure of 1, 3-dimethylimidazolium salts. J. Phys. C 15, S159-S166 (2003).

40 Osaka, T., Komaba, S., Uchida, Y., Kitahara, M., Momma, T \& Eda, N. Performance of a lithium metal anode in poly(vinylidene fluoride)-type gel electrolyte. Electrochem. Solid State Lett. 2, 215-217 (1999).

41 Ismail, I., Noda, A., Nishimoto, A. \& Watanabe, M. XPS Study of lithium surface after contact with lithium-salt doped polymer electrolytes. Elecrochim. Acta 46, 1595-1603 (2001).

42 Zhang, H., Kulkarni, S. \& Wunder, S. L. Blends of POSS-PEO $(n=4) 8$ and high molecular weight poly(ethylene oxide) as solid polymer electrolytes for lithium batteries. J. Phys. Chem. B, 111, 3583-3590 (2007).

43 Vogel, H. The temperature dependence law of the viscosity of fluids. Phys. Z 22, 645-646 (1921).

44 Fulcher, G. S. Analysis of recent measurements of the viscosity of glasses. J. Am. Ceram. Soc. 8, 339 (1925).

45 Tamman, G. \& Hesse, W. Z. Die Abhängigkeit der Viscosität von der Temperatur bie unterkühlten FlüssigkeitenAnorg. Allg. Chem. 156, 245-257 (1926). 